\title{
Pharyngitis and COVID-19 infection
}

Mohammad Hossein Zamanian', Zeinab Mohseni Afshar ${ }^{1, *}$, Arefeh Babazadeh², Soheil Ebrahimpour ${ }^{2}$

${ }^{1}$ Clinical Research Development Center, Imam Reza Hospital, Kermanshah University of Medical Sciences, Kermanshah, I.R. Iran

${ }^{2}$ Infectious Diseases and Tropical Medicine Research Center, Health Research Institute, Babol University of Medical Sciences, Babol, I.R. Iran

\section{To the Editor}

Severe acute respiratory syndrome coronavirus 2 (SARSCoV-2) causing novel coronavirus disease 2019 (COVID-19) has quickly evolved from an epidemic outbreak in Wuhan, Hubei Province, China, into a pandemic (1). Subsequently, clinicians are facing several challenges when considering cases with suspected COVID-19. Presentations of COVID-19 have ranged from asymptomatic to severe illness leading to death.

Mild symptoms of disease comprise fever, cough, lethargy, myalgia, headache and sore throat (2). Sore throat, which is the result of pharyngitis, is a quite common reported complaint, similar to every viral infection. Up to the present time, almost all cases of COVID-19 induced pharyngitis have been of non-exudative types; however, recently the opposite has been observed.

In our outpatient clinic in Kermanshah, Iran, we confronted some cases of exudative pharyngitis due to COVID-19 infection. Six patients with COVID-19 (one male, five females), ranging in age from 30 years to 55 years, presented with the chief complaint of fever and sore throat, without any obvious respiratory symptoms. Three patients experienced episodes of diarrhoea in the past recent days. In the physical examination, exudative pharyngitis was evident (Figure 1), without any cervical lymphadenopathy. Two cases presented conjunctivitis. Arterial blood oxygen saturation was $>95 \%$ in ambient air in all of these patients. Lymphopenia was detected in four cases. Due to their recent close contact with COVID-19 patients, their nasopharyngeal specimens were collected, which were positive by real-time reverse-transcriptase-polymerase chain reaction (RT-PCR). Interestingly, these patients did not have any radiological findings consistent with COVID-19 infection. Considering the COVID-19 epidemic and their positive molecular tests, all of these patients were started on a treatment regimen with hydroxychloroquine, which resulted in a rapid improvement

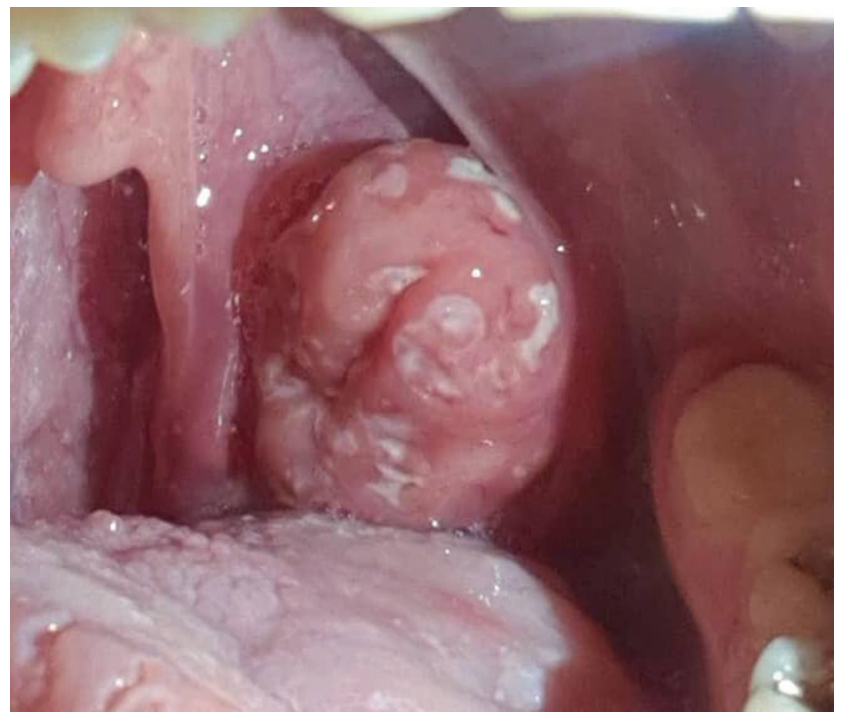

Figure 1. Patient with exudative pharyngitis.

in the follow-up visit. The interesting question has arisen exudative pharyngitis that might be caused by bacteria such as group A Streptococcus (GAS) bacteria. According to the Modified Centor Score and culture management approach to pharyngitis, the total score was 1 in two cases and the others got a total score of 2 (3). Patients with a total score of 1 did not need further evaluation since viral origin was defined in terms of their symptoms. However, for patients with a total score of 2, pharyngeal swabs for Streptococcus culture should be obtained. Unfortunately, the four patients did not consent to perform throat culture; therefore, we prescribed antiviral drugs and antimicrobial agents simultaneously. Based on our findings and review of current literature, we could consider that a number of COVID-19 patients presented

*Corresponding author: Zeinab Mohseni Afshar

E-mail: baboldr2019@gmail.com.Tel: +989111156511

ว Open Access. (c) 2021 Zamanian et al., published by Sciendo

(c) BY-NC-ND This work is licensed under the Creative Commons Attribution-NonCommercial-NoDerivs 4.0 License. 
with an atypical manifestation of the infection, although in a low percentage. We report, for the first time, some cases of exudative pharyngitis due to COVID-19 infection. It is important to note that although fever and respiratory involvement are typical of COVID-19 infection, other manifestations should not be neglected during this pandemic and special consideration is important to establish the diagnosis and be able to establish early preventive measures. More studies are needed to confirm and better understand atypical.

\section{Conflicts of interest}

All authors declare no conflict of interest.

\section{References}

1. Whitworth J. COVID-19: a fast evolving pandemic. Transactions of the Royal Society of Tropical Medicine and Hygiene. 2020;114(4): 241-248.

2. Javanian M, Bayani M, Shokri M, Sadeghi-Haddad-Zavareh $M$, Babazadeh A, Yeganeh B, et al. Clinical and laboratory findings from patients with COVID-19 pneumonia in Babol North of Iran: a retrospective cohort study. Romanian Journal of Internal Medicine. 2020;58(3): 161-167.

3. Choby BA. Diagnosis and treatment of streptococcal pharyngitis. American Family Physician. 2009;79(5): 383-390. 